\title{
Solubilization of Phospholipid Bilayer Caused by Surfactants
}

\author{
A. de la Maza* and J.L. Parra Juez \\ Instituto de Tecnologia Química y Textil, Consejo Superior de Investigaciones Cientificas (C.S.I.C.), Departamento de Tecnologia \\ Química, 08034 Barcelona, Spain
}

\begin{abstract}
The interaction of surfactants with liposomes eventually leads to the rupture of such structures and the solubilization of the phospholipid components. In this paper, solubilization is regarded as a decrease in light scattering of liposome suspensions. To this end, in accordance with the nomenclature, adopted by Lichtenberg, three parameters were considered as corresponding to the effective surfactantllipid molar ratios (Re)at which light scattering starts to decrease, $\mathbf{R} \mathbf{e}_{\text {sat }}$; reaches $50 \%$ of the original value, $\mathbf{R} \mathbf{e}_{50}$; and shows no further decrease, $\mathbf{R e}_{\text {sol }}$. These parameters corresponded to the Re at which the surfactant (i) saturated the liposomes, (ii) resulted in a 50\% solubilization of vesicles and (iii) led to a total solubilization of liposomes. The surfactants tested were the nonionicsurfactant octylphenol ethoxylated with 10 units of ethylene oxide or Triton X-100 (OP-10EO), two anionic surfactants, sodium dodecyl sulfate and sodium dodecyl ether sulfate, and an amphotericsurfactant dodecyl betaine (D-Bet). Unilamellar liposomes formed by egg phosphatidylcholine containing increasing amounts of phosphatidic acid were used. The Re parameters were the lowest for D-Bet, followed by OP-10EO, whereas the anionic surfactants always showed the highest values regardless of the electrical charge of the lipid bilayers. These parameters seem also to be inversely related to the critical micelle concentration (CMC) of the surfactant, except for OP-10EO. Moreover, the CMC values of the surfactantllipid systems at $0.5 \mathrm{mM}$ lipid concentration corresponded in all cases to the surfactant concentration at which liposomes were saturated by surfactants. As a consequence, this ratio can be regarded as an interesting parameter associated with the mixed micelle formation in liposome solubilization.
\end{abstract}

KEY WORDS: Critical micelle concentration, light scattering changes, liposome solubilization, surface tension changes.

Liposomes are lipid-water systems widely used as sirnplified models of different biological membranes. The study of the physicochemical process involved in liposomesurfactant interactions has been of great interest because this can provide useful information about the complex phenomenon of the solubilizing interactions between phospholipids and surfactants (1-4).

A significant contribution has been made by Lichtenberg (5), who postulated that the minimum effective surfactantllipid ratio producing solubilization depends on the surfactant critical micelle concentration (CMC) and on the bilayerlaqueous medium partition coefficients, rather than on the nature of the surfactants. Accordingly, we carried out studies on the partition coefficients of different surfactants (6)to determine the main factors involved in the modifications of the permeability of lipid bilayers by different amphiphilic molecules.

In the present work, we have attempted to characterize the solubilization of electronegatively charged unilamellar

*To whom correspondence should be addressed at Instituto de Tecnología Química y Textil, Consejo Superior de Investigaciones Cientificas (C.S.I.C.)C/Jorge Girona, 18-26, 08034 Barcelona, Spain. lipid bilayers by surfactants. Solubilization was assessed as a decrease in light scattering $(7,8)$ and surface tension of the liposome/surfactant systems during the solubilizing process. To evaluate the iight scattering variations, three parameters were determined, namely effective surfactantllipid molar ratios (Re)at which light scattering starts to decrease $(\operatorname{Re}$, ); reaches $50 \%$ of the original value $(\operatorname{Re},$,$) ; and shows$ no further decrease (Re,,), according to the nomenclature adopted by Lichtenberg $(5,9)$.

The selected surfactants were sodium dodecyl sulfate (SDS)as a typical anionic surfactant; sodium dodecyl ether sulfate (SDES) to assess the influence of the ethylene oxide groups on the anionic surfactant's behavior; octyl-phenol polyethoxylated with 10 ethylene oxide units, Triton X-100, (OP-10EO) as a representative nonionic surfactant, which is widely used in membrane studies (10-12) and dodecyl betaine (D-Bet)as a representative of amphoteric surfactants (13).

Some of the results obtained in this study will provide information on physicochemical factors involved in the interactions of surfactants with lipid bilayers and on the way they affect vesicle solubilization. This information also allowed us to establish a criterion for the evaluation of surfactant activity on phospholipid vesicles.

\section{EXPERIMENTAL PROCEDURES}

Materials. SDS was obtained from Merck (Darmstadt, Germany) and further purified by a column chromatographie method (14).SDES was supplied by Tenecco SA (Barcelona,Spain). The latter was a commercial-gradeproduct with an active matter of $28.8 \%$ with a 2.5 average in EO units and the following mix in alkyl chain: C-10, $3.9 \%$; $-12,68.1 \%$; C-14, $22.2 \%$ and C-16, 4.9\%. OP-10EO was purchased from Fbhm and Haas (Paris, France) and had an active matter content of $100 \%$. The amphoteric surfactant D-Bet was especially prepared by Albright and Wilson, Ltd. (Warley, West Midlands, United Kingdom); the active matter was $30 \%$ in aqueous solution and the amino free content was $0.20 \%$. Phosphatidic acid (PA) from egg yolk lecithin was purchased from Sigma Chernical Co. (St. Louis, MO). Phosphatidylcholine (PC) was purified from egg lecithin (Merck)according to the method of Singleton et al. (15)and was pure by thin-layer chromatography (TLC).Piperazine-1,4 bis(2-ethanesulfonic acid) (PIPES buffer) was obtained from Merck. The buffer used was $20 \mathrm{mM}$ PIPES, adjusted to $\mathrm{pH} 7.2$ with $\mathrm{NaOH}$, supplemented with $110 \mathrm{mM} \mathrm{Na} \mathrm{SO}_{4}$. Water was purified by the Milli-Ro system (Millipore,Millford, MA). Polycarbonate membranes and membrane holders were purchased from Nucleopore (Pleasanton, CA).

Liposome preparation. Unilamellar liposome vesicles of a defined size (about $100 \mathrm{~nm}$ ) were prepared by extrusion of large unilamellar vesicles previously obtained by the reverse-phase evaporation method $(16,17)$ based on an earlier one described by Szoka and Papahadjopoulos (18). A lipidic film was formed by removing the organic solvent by rotatory evaporation from chloroform solutions of 
lipids (lipidcompositions PCIPA 9:1 and 8:2 molar ratio). The lipids were then redissolved in diethyl ether, and the PIPES buffer was added to the solution of phosholipids. Gentle sonication led to the formation of a waterloil(W/O)type emulsion. After evaporating the ethyl ether under reduced pressure, a viscous gel was formed. The elimination of the final traces of the organic solvent transformed the gel into a liposome suspension. Unilamellar vesicles were obtained by extrusion of vesicle suspensions through $800,400,200$ and $100 \mathrm{~nm}$ polycarbonate membranes to achieve a uniform size distribution (19).The phospholipid concentration range in liposome suspensions studied was $0.50-5.0 \mathrm{mM}$.

Phosphorus estimation Phospholipidconcentrations of the liposome vesicles were determined by the ascorbic acid spectrophotometric method for total phosphorus estimation (20).

Determination ofparticle size distribution and stability of liposome preparations. Mean size and polydispersity of the liposome preparations were determined by a photon correlator spectrometer (Malvern Autosizer 4700c PS/MV; Malvern, England). Particle size distributions were established by particle number measurements. Samples were adjusted to the adequate concentration range with PIPES buffer, and the measurements were taken at $25^{\circ} \mathrm{C}$ at a lecture angle of $90^{\circ}$. The particle size distribution of the liposome suspensions after preparation (phospholipidconcentration ranging from 0.5 to $5.0 \mathrm{mM}$ ) varied little (around $100 \mathrm{~nm}$ ). The polydispersity indexes were lower than 0.1 , indicating that the distributions were homogeneous. Likewise, the particle size distribution of liposomes after addition of equal volumes of PIPES buffer and equilibra. tion for $24 \mathrm{~h}$ at $25^{\circ} \mathrm{C}$ showed in all cases similar values as those obtained after preparation, with a slight increase in the polydispersity index (from 0.12 to 0.15 ). As a consequence, the liposome preparations appeared to be stable in absence of surfactants under the experimental conditions used in liposome solubilization studies.

Liposome solubilization by surfactants. The perturbation produced by the surfactants in the phospholipid bilayers leads to the solubilization of the lipid components via mixed micelle formation (5).This solubilization results in changes in light scattering of these systems, which depends on the nature of both surfactant and lipid components. This can be monitored by measuring the variations in light scattering during the solubilizing process (8).

To evaluate the variations obtained with the various surfactants and bilayer compositions, the Re in an aggregate (liposome or micelle) is defined (9)in Equation 1.

$$
\operatorname{Re}=\frac{[\text { total surfactant }]-[\text { surfactant monomer }]}{[\text { total phospholipid }]-[\text { phospholipid monomer }]}
$$

The second term of the denominator is negligible due to the low solubility of phospholipids in water.

The overall solubilization process of phospholipids by surfactants can be characterized by three parameters termed $\mathrm{Re},, \mathrm{Re}_{50 \%}$ and $\mathrm{Re},,$, according to the nomenclature adopted by Lichtenberg $(5,9)$, corresponding to the Re at which light scattering starts to decrease, reaches $50 \%$ of the original value and shows no further decrease. These parameters correspond to the Re at which surfac- tant (i) saturates the liposomes, (ii) results in a $50 \%$ solubilization of vesicles and (iii)leads to total solubilization of the liposomes.

These parameters can be determined from the linear dependence existing between the surfactant concentrations required to achieve these parameters and the phospholipid concentration in liposomes. The equations describing the necessary surfactant concentration needed to saturate the bilayer (Eq. 2), to solubilize $50 \%$ of the liposomes (Eq. 3) or to achieve the complete solubilization of liposomes via mixed micelle formation (Eq.4) are given as:

$$
\begin{aligned}
\mathrm{S}_{\mathrm{sat}} & =\mathrm{S}_{\mathrm{a}}+\operatorname{Re}_{\mathrm{sat}} \times(\mathrm{PL}) \\
\mathrm{S}_{50 \%} & =\mathrm{S}_{\mathrm{b}}+\mathrm{Re}_{50 \%} \times(\mathrm{PL}) \\
\mathrm{S}_{\mathrm{sol}} & =\mathrm{S}_{\mathrm{c}}+\mathrm{Re}_{\mathrm{sol}} \times(\mathrm{PL})
\end{aligned}
$$

where $\mathbf{S}_{\text {sat }}, \mathbf{S}_{50 \%}$ and $\mathbf{S}_{\text {sol }}$ are the total surfactant concentrations ( $\mathrm{mM}$ ), and (PL)is the phospholipid concentration (mM) in liposomes. The effective surfactant-to-phospholipid molar ratios $\left(\operatorname{Re}_{,,,}, \operatorname{Re}_{50 \%}\right.$ and $\left.\mathrm{Re}, \ldots\right)$ and the aqueous concentrations of surfactants $\left(\mathbf{S}_{\mathrm{a}}, \mathbf{S}_{\mathrm{b}}\right.$ and $\left.\mathbf{S}_{\mathrm{c}}\right)$ are in each curve, respectively, the slope and the ordinate at the origin (zero phospholipid concentration).

Liposome suspensions were adjusted to the adequate lipid concentration (from 1.0 to $10.0 \mathrm{mM}$ ). To these, equal volumes of the proper surfactant solutions were added, and the resulting mixtures were left to equilibrate for 24 h. Light scattering measurements were made at $25^{\circ} \mathrm{C}$ with a Shimadzu RF-540 spectrofluorophotometer equipped with a thermoregulated cell compartment (Kyoto,Japan) with both monochromators adjusted to $500 \mathrm{~nm}$. The assays were carried out in triplicate, and the results given are the average of those obtained.

Surface tension measurements. Surface tensions of buffered solutions of single surfactants and of liposome/surfactant systems were measured by the ring method (21) at $25^{\circ} \mathrm{C}$ with a Krüss (Hamburg, Germany) tensiometer (processor tensiometer K-12), which determines directly the real surface tension values at equilibrium.

CMC determination. The CMC for a single surfactant or the different liposomelsurfactant systems at a lipid concentration of $0.5 \mathrm{mM}$ in PIPES buffer were determined at $25^{\circ} \mathrm{C}$ by plotting the surface tension values $v$ s. surfactant concentration.

\section{RESULTS AND DISCUSSION}

Solubilization studies. The solubilization of liposomes by surfactants was studied by monitoring the variations in the light scattered by the surfactantlliposome systems as a function of surfactant concentration. In this work, lipid bilayers consisted of PC unilamellar vesicles, to which PA was added, yielding liposomes with molar ratios PCIPA of $9: 1$ and $8: 2$, to increase the negative charge of the bilayers.

Figure 1 shows the solubilizationcurves of the liposome preparations (PCIPA 9:1 molar ratio and lipid concentration from $0.5 \mathrm{mM}$ to $5.0 \mathrm{mM}$ ) arising from the addition of different concentrations of nonionic surfactant OP. 10EO. The increased light-scattering values obtained 


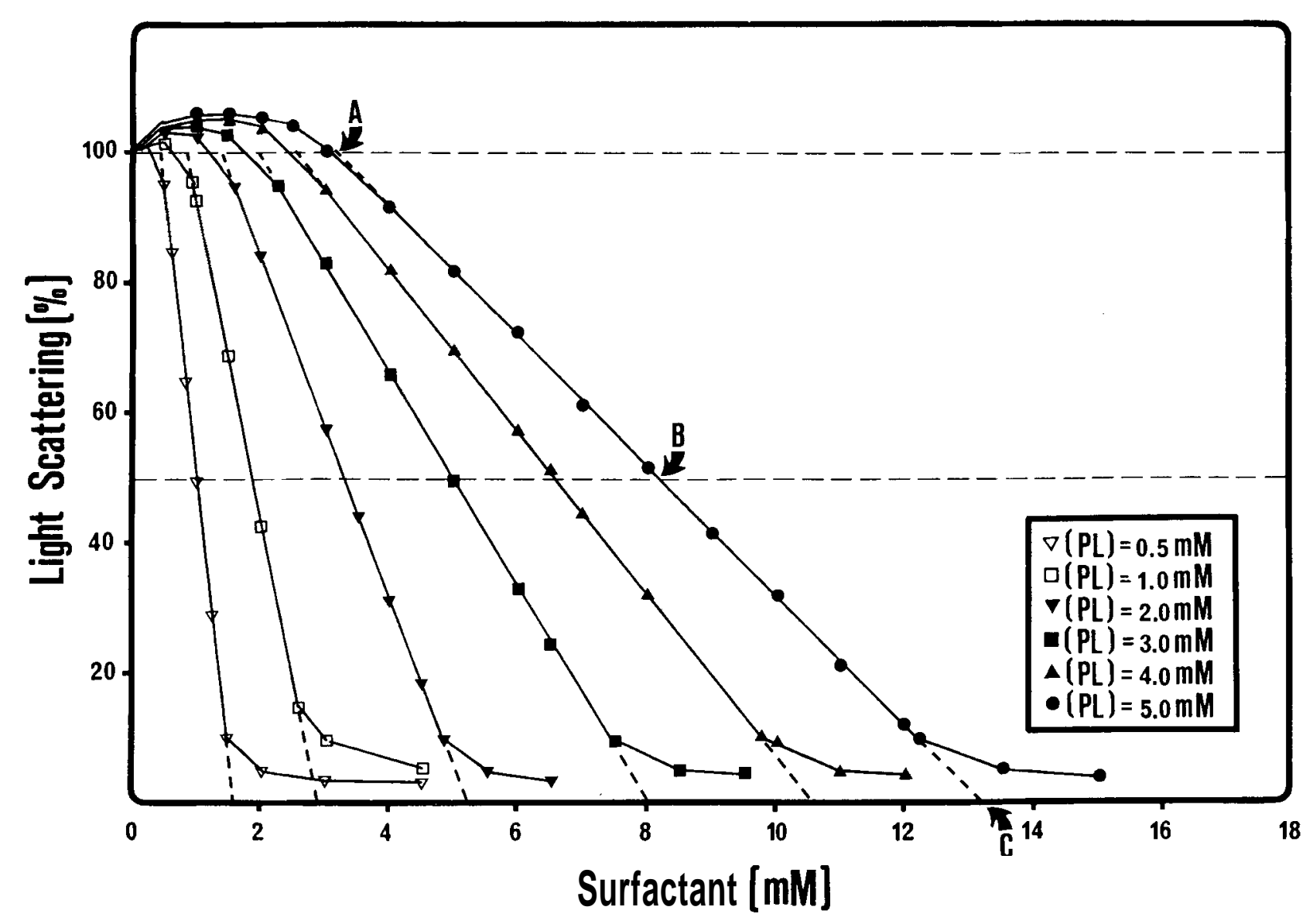

FIG. 1. Percentage change in light scattering of unilamellar liposomes [phosphatidylcholinelphosphatidic acid (PCIPA)9:1 molar ratio] at bilayer lipid concentrationsranging between 0.5 and $5.0 \mathrm{mM}$ us. nonionic surfactant octyl-phenolethoxylated with 10 units of ethylene oxide or Triton X-100 surfactant concentration. Abbreviation: PL, phospholipids.

upon addition of low levels of surfactant can be explained by bearing in mind that low amounts of surfactant incorporated into bilayers increase the size of vesicles $(5,8,10)$. From these curves, the surfactant concentrations that produce saturation, half solubilization and total solubilization of the liposomes can be obtained by graphical methods. The arrows A, B and C (curve for $5.0 \mathrm{mM}$ lipid concentration) correspond to these respective parameters, i.e, the surfactant concentration at which light scattering starts to decrease $\left(\mathrm{S}_{\mathrm{sat}}\right)$, reaches $50 \%\left(\mathrm{~S}_{50 \%}\right)$ and shows no further decrease $\left(\mathbf{S}_{\mathrm{sol}}\right)$. Similar curves (not shown) were obtained for the different surfactants tested.

The measured surfactant concentrations are plotted $v s$. phospholipid concentration (PCIPA 9:1 molar ratio)(Figs. 2-4). An acceptable linear relationship is established in each case. The straight lines obtained correspond to the aforementioned equations (Eqs. 2,3 and 4) where the Re parameters and the aqueous concentration of surfactants are for each curve, respectively, the slope and the ordinate at the origin (zerophospholipid concentration). Similar results were obtained when treating more negatively charged liposomes (PCIPA8:2 molar ratio) with these surfactants under the same conditions (curves not shown). The solubilizing parameters obtained, including the regression coefficients of the straight lines (Figs. 2-4), and the CMC values of the surfactants in the buffered medium are shown in Table 1.

In the vast majority of cases, solubilization of bilayers is only slightly affected by the presence of increased PA in the lipid bilayers. In that case, liposomes appear to be slightly more resistant to surfactant solubilization, except for the amphoteric D-Bet surfactant. Bearing in mind that lipid bilayers are electronegatively charged, the possible electrostatic repulsion between the charged bilayers and the anionic surfactants (SDS and SDES) could affect the Re parameters, resulting in slightly increased values. These electrostatic forces could also affect the Re parameters of the amphoteric surfactant D-Bet, which under these conditions ( $\mathrm{pH} 7.20$ ) shows a cationic character (22). However, our results confirm the small influence of the increased electrostatic forces (PCIPA 9:1 and 8:2 molar ratios) on the Re parameters for each surfactant tested (8).

The surfactant concentrations in the aqueous medium were always similar or higher than the corresponding CMCs regardless of the electrical charge of the liposomes. The results suggest that surfactant-liposome solubilization is mainly determined by the formation of mixed micelles formed by the surfactant and the phospholipid molecules, unlike the behavior of these surfactants in sub. 


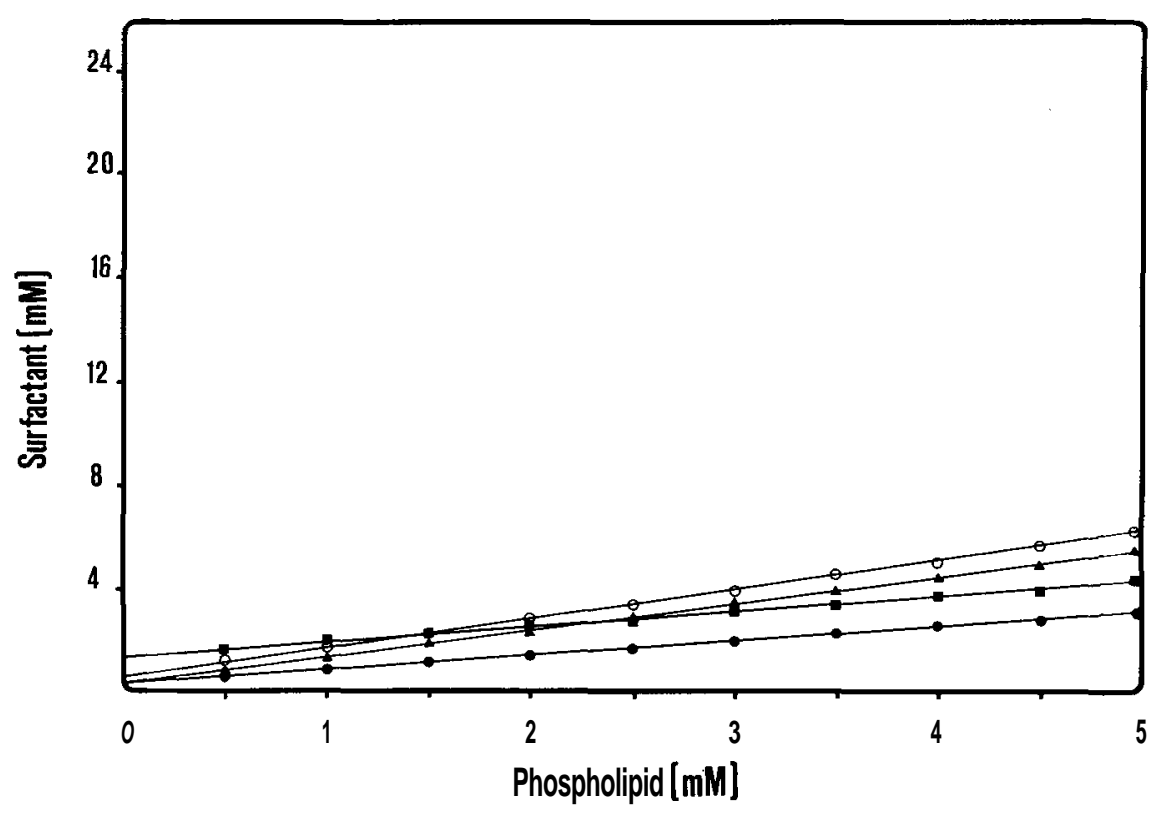

FIG. 2. Plots of the concentrations of amphoteric surfactant dodecyl betaine (D-Bet)( $\mathbf{D})$, sodium dodecyl sulfate (SDS) (O), sodium dodecyl ether sulfate (SDES)(A)and nonionic surfactant octyl-phenol ethoxylated with 10 units of ethylene oxide or Triton X-100 (OP-10EO) ( ), corresponding to surfactant concentration at which liposomes were saturated by surfactant for liposomes (PCIPA 9:1 molar ratio), us. bilayer lipid concentration. See Figure 1 for abbreviations.

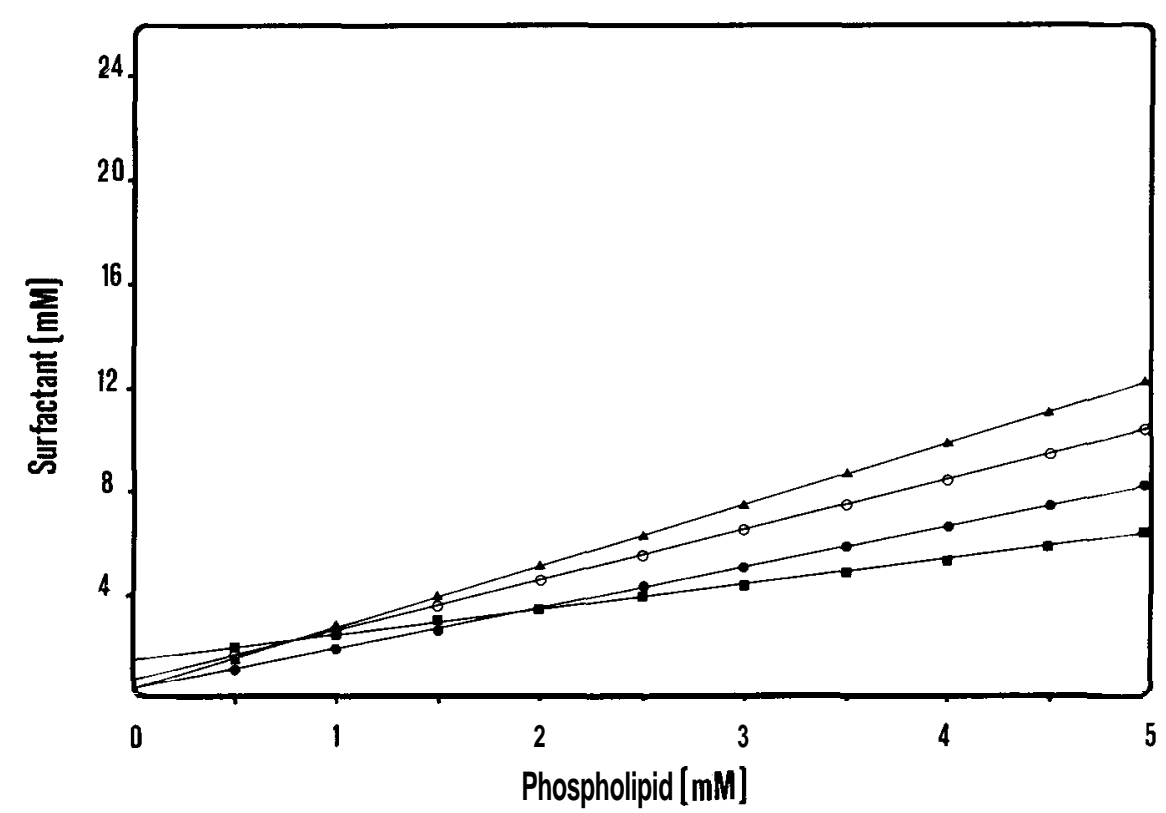

FIG. 3. Plots of the concentrations of D-Bet (a), SDS (O), SDES (A) and OP-10EO ( $\bullet$ ), corresponding to surfactant concentration at which liposomes were solubilized $50 \%$ for liposomes (PCIPA 9:1 molar ratio) us. bilayer concentration. See Figure 2 for abbreviations. 


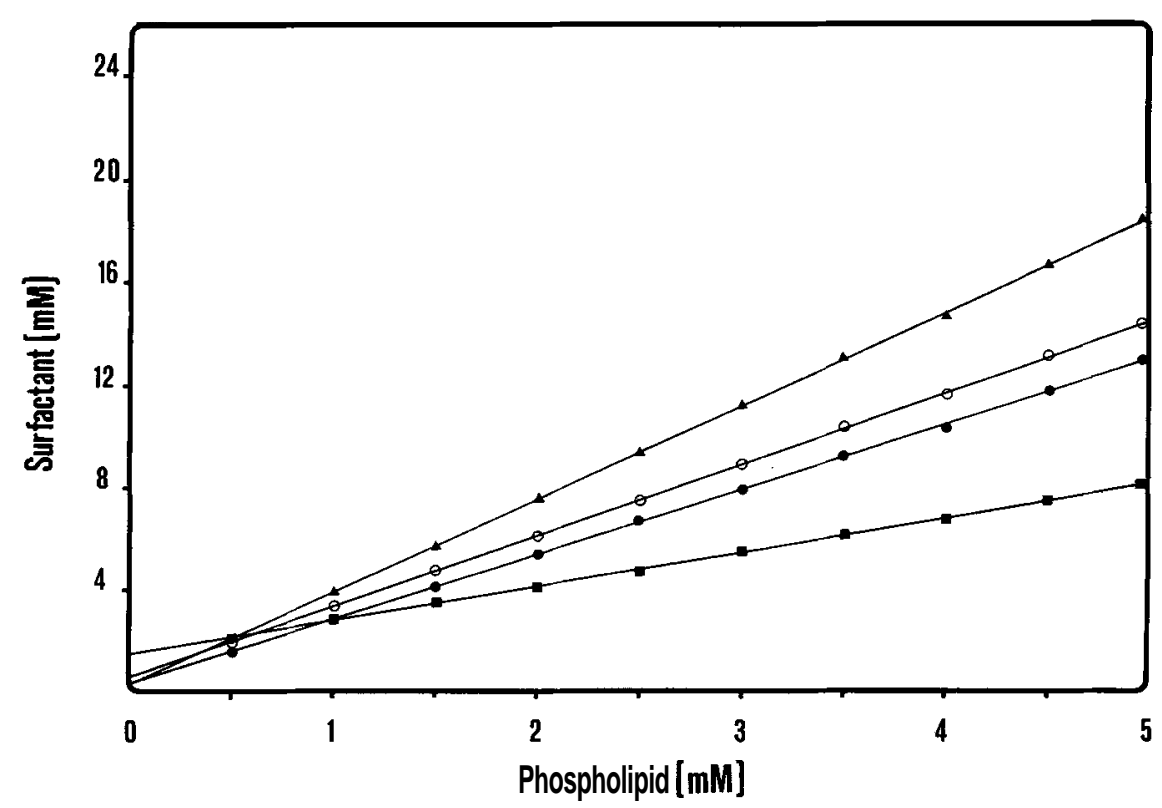

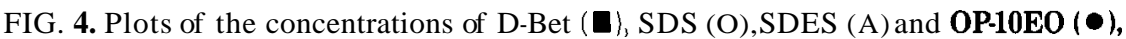
corresponding to surfactant concentration at which liposomes achieved complete solubilization for liposomes (PCIPA 9:1 molar ratio) us. bilayer lipid concentration. See Figure 2 for abbreviations.

\section{TABLE 1}

Solubilizing Parameters of Liposomes (PCIPA 9:1 and 8:1 molar ratios): Bilayer Lipid Composition. The CMC of Surfactants and the Regression Coefficients of the Straight Lines of Figures 2-4 Are Also Included ${ }^{\mathrm{a}}$

\begin{tabular}{|c|c|c|c|c|c|c|c|c|}
\hline & \multirow{2}{*}{$\begin{array}{l}\mathrm{CMC} \\
(\mathrm{mM})\end{array}$} & \multicolumn{7}{|c|}{ PCIPA $(9: 1)$} \\
\hline & & $\overline{S_{a}}$ & $\overline{S_{b}}$ & $\mathrm{~S}_{\mathrm{c}}$ & $R e_{\text {sat }}$ & $\operatorname{Re}_{50 \%}$ & $\mathrm{Re}_{\mathrm{sol}}$ & $\mathrm{r}^{2}$ \\
\hline $\mathrm{D}-\mathrm{B}$ & 1.25 & 1.25 & 1.27 & 1.30 & 060 & 1.00 & 1.40 & 0.996 \\
\hline $\mathrm{SD}$ & 0.50 & 0 & 0.51 & 0.52 & & 1.99 & & 992 \\
\hline SDES & 0.12 & 0.12 & 0.13 & 0.14 & 1. & 2.40 & 3.70 & 0.996 \\
\hline \multirow[t]{3}{*}{ OP-10EO } & 0.15 & 0.18 & 0.21 & 0.24 & 0.60 & 1.60 & 2.60 & 0.998 \\
\hline & & \multicolumn{7}{|c|}{ PCIPA $(8: 2)$} \\
\hline & & $\mathrm{S}_{\mathrm{a}}$ & $S_{b}$ & $S_{c}$ & $R e_{\text {sat }}$ & $\mathrm{Re}_{50 \%}$ & $\mathrm{Re}_{\mathrm{sol}}$ & $\mathbf{r}^{2}$ \\
\hline $\mathrm{P}$ & & $\overline{1.25}$ & 1.26 & 1.30 & $\overline{0}$ & 0.9 & 130 & $\overline{90}$ \\
\hline & & 0.50 & 0.52 & 0.53 & & 2.08 & 2.90 & \\
\hline SDES & 0.1 & 0.14 & 0.15 & 0.14 & 1.22 & 2.50 & 3.90 & 0.99 \\
\hline OP-10EO & 0.15 & 0.17 & 0.19 & 0.22 & 0.68 & 1.64 & 2.70 & 0.99 \\
\hline
\end{tabular}

${ }^{a}$ Abbreviations: PC, phosphatidylcholine; PA, phosphatidic acid; CMC, critical micelle concentration; D-Bet, amphoteric surfactant dodecyl betaine; SDS, sodium dodecyl sulfate; SDES, sodium dodecyl ether sulfate; OP-10EO, nonionic surfactant octyl-phenol ethoxylated with 10 units of ethylene oxide or Triton X-100. Re, effective surfactantllipid molar ratio at which light scattering starts to decrease $\left(\mathrm{Re}_{\text {sat }}\right)$, reaches $50 \%$ of the original value $\left(\mathrm{Re}_{50 \%}\right)$, and shows no further decrease $(\operatorname{Re},$,$) .$

solubilizing processes in which the action of surfactant monomers play an important role (6).

In terms of the Re, the amphoteric surfactant D-Bet shows the lowest values (especiallyfor PCIPA 8:2 liposome lipid compositions) followed by the nonionic surfactant OP-10EO, whereas the anionic surfactants always show the highest values, particularly SDES, regardless of the electrical charge of the liposomes.
Comparing the Re values of the two anionic surfactants, the slight decrease of $\mathrm{Re}_{\mathrm{sat}}$ and the increase of the $\mathrm{Re}_{50 \%}$ and Re,., parameters of the SDES compared to SDS could be attributable to the presence of $2.5 \mathrm{EO}$ units in its molecular structure, because it is the only structural difference existing between these anionic surfactants. This raises questions about the influence of the $\mathrm{EO}$ in bilayer saturation and solubilization of these surfactants. Those EO units increase the hydrophilic character of the surfactants. This fact could be responsible for the changes in the Re parameters because it reduces the degree of irritation and the CMC value (23), thus increasing water solubility (24).

The data in Table 1 also reveal that the Re parameters appear to be inversely correlated with the CMC of the surfactants tested in the working medium. Thus, the amphoteric surfactant D-Bet, which has the highest CMC value $(1.25 \mathrm{mM})$ presents the lowest Re parameters, whereas the SDES surfactant (CMC0.12 mM) shows the highest values, regardless of the electrical charge of liposomes. This tendency is also observed for the anionic surfactants, except for the Re,,, parameter. However, an exception is detected for OP-10EO, which has a low CMC value $(0.15$ $\mathrm{mM}$ ) but appears to be particularly effective to bilayer saturation and solubilization. The Re parameters obtained for this nonionic surfactant are comparable with those reported in the literature (2), confirming the effectiveness of this surfactant in the interaction with lipid bilayers.

Surface tension studies. To establish the relationship between the Re parameters and the CMC of the surfactants tested, a systematic investigation of surface tension was carried out by comparing the surface tension values of the single surfactants and the surfactantlliposome systems us. surfactant concentration. Figure 5 plots the surface tension variation $\boldsymbol{u s}$. surfactant concentration 


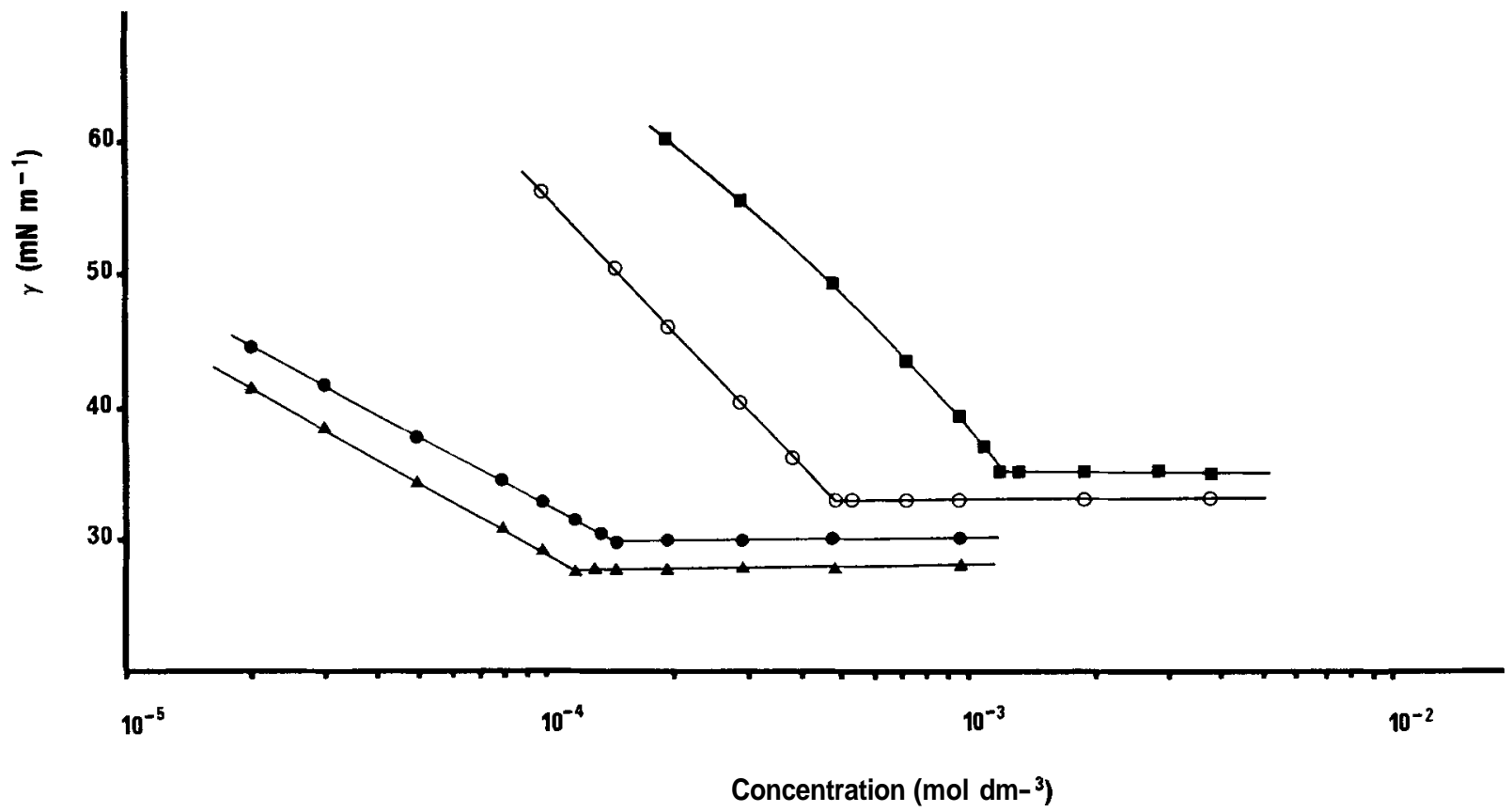

FIG. 5. Plots of the surface tensions of D-Bet ( $\mathbf{\square})$, SDS (O), SDES (A)and OP-10EO ( $\bullet$ ). (PCIPA 9:1 molar ratio and lipid concentration $0.5 \mathrm{mM}$ ) us. surfactant concentration in piperazine-1,4 bis(2-ethane-sulfonic acid) (PIPES) buffer. See Figures 1 and 2 for abbreviations.

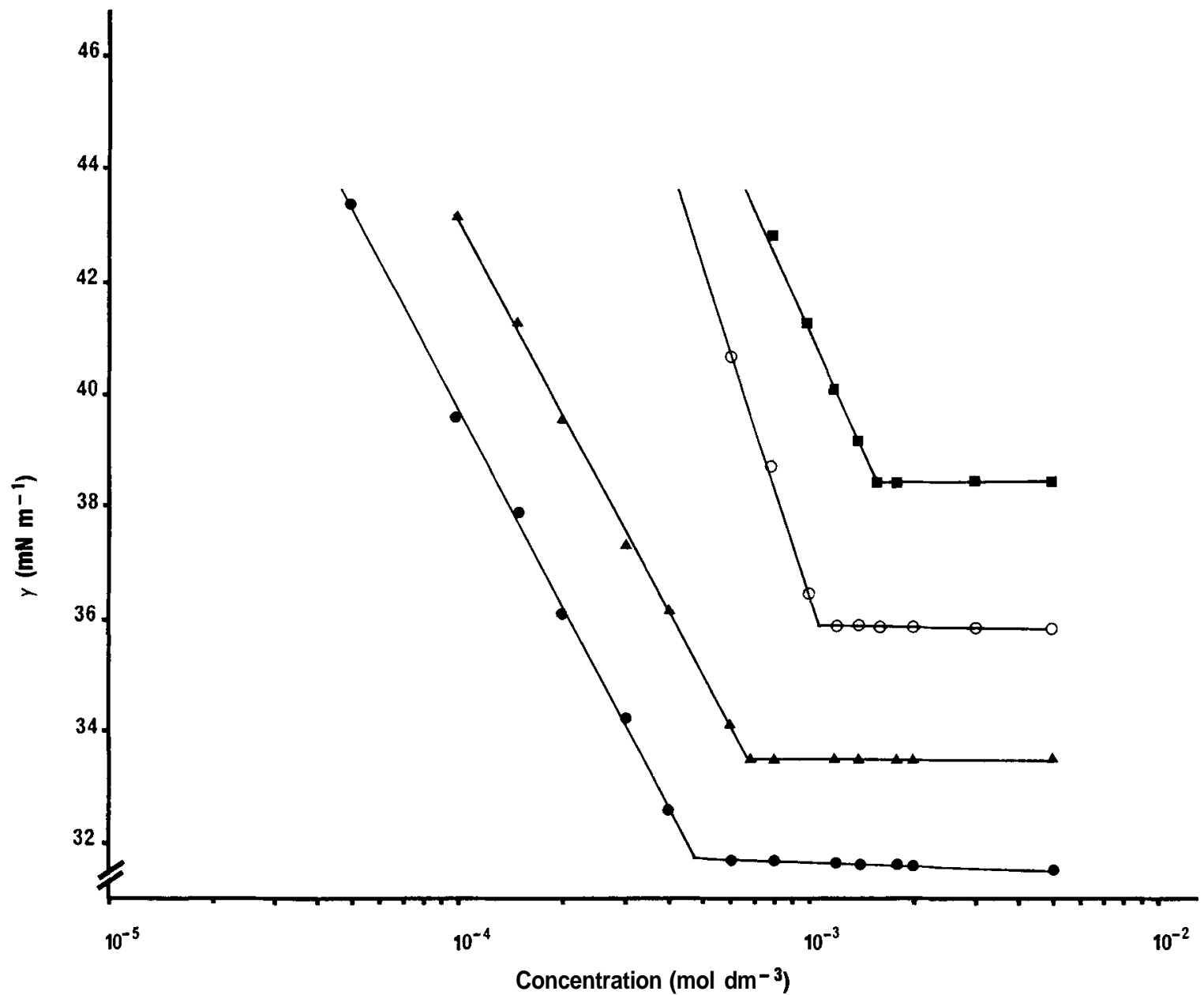

FIG. 6. Plots of the surface tensions [D-Bet (a), SDS (O),SDES (A) and OP-10EO ( $)$ ] of liposomelsurfactant systems for unilamellar liposomes with the same surfactants (PCIPA 9:1 molar ratio and lipid concentration $0.5 \mathrm{mM}$ ) us. surfactant concentration in PIPES buffer. See Figures 1, 2 and 5 for abbreviations. 


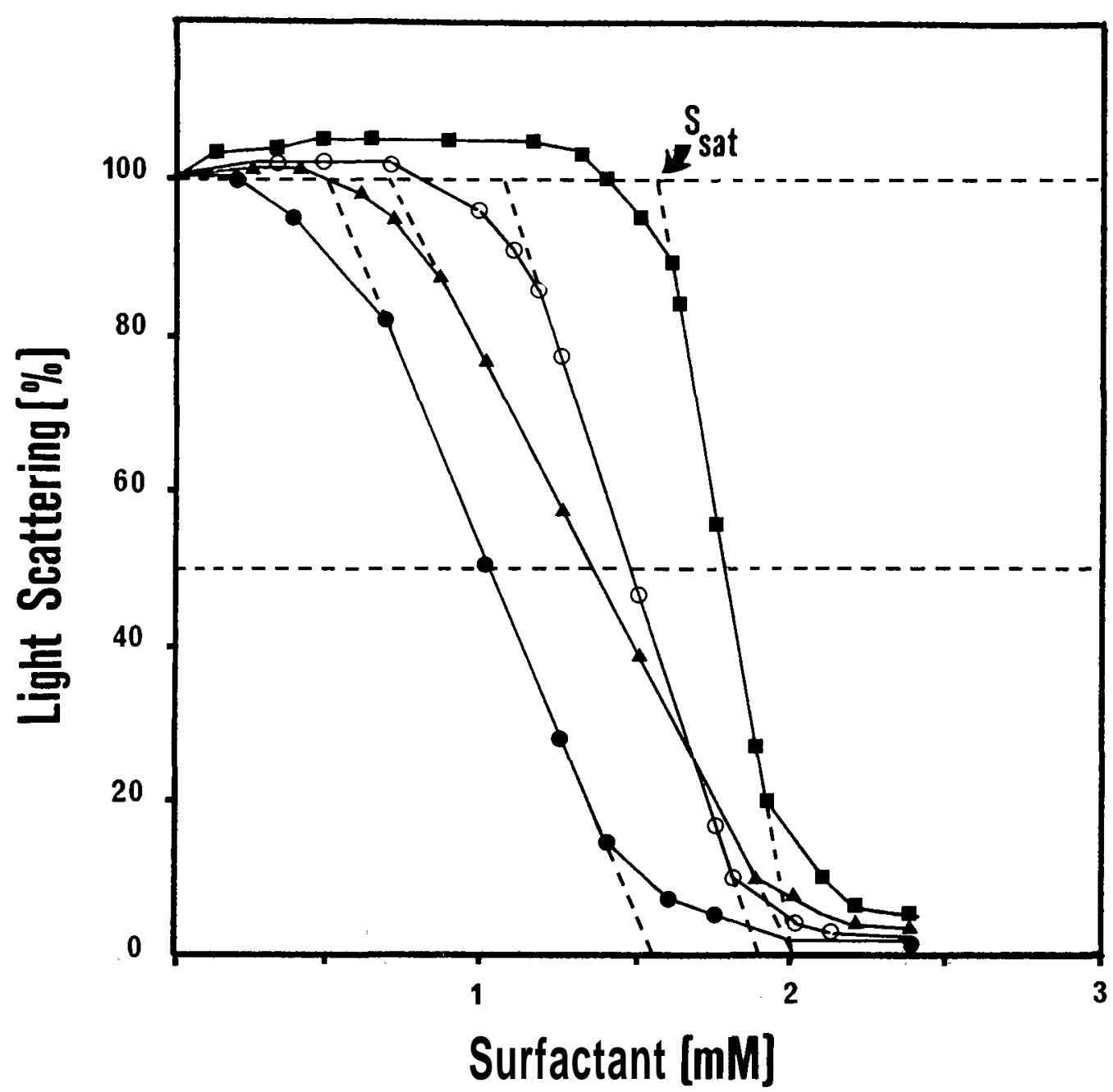

FIG. 7. Percentage change in light scattering of liposomes (PCIPA 9:1 molar ratio, lipid concentration 0.5 $\mathrm{mM})$ us. surfactant concentration in the presence of D-Bet $(\square)$, SDS $(O)$, SDES (A) and OP-10EO $(\bullet)$ surfactants. See Figures 1 and 2 for abbreviations.

for D-Bet, SDS, SDES and OP-10EO and shows the conventional inflexion at their CMC values. Figure 6 shows the same variation for liposome/surfactant systems (lipid concentration $0.5 \mathrm{mM}$ and PCIPA molar ratio 9:1) vs. surfactant concentration. In this figure, surface tension values decrease with increasing surfactant concentration and also present inflection points. These points can be considered physicochemical parameters related to the CMC of the surfactantlphospholipid binary systems. In the present work, this inflection point is expressed as the $\mathrm{CMC}_{\text {system. }}$ Similar physicochemical behavior was observed when treating more negatively charged liposomes (PC/PA molar ratio 8:2) with the surfactants under the same conditions.

Comparing the $\mathrm{CMC}$ and $\mathrm{CMC}_{\text {system }}$ values (Figs. 5 and 6) shows that the presence of lipid bilayers in the aqueous medium requires an increased surfactant concentration to achieve the corresponding $\mathrm{CMC}_{\text {system }}$. The displacements can be attributed to interaction of the components that leads to solubilization of the system. Likewise, in all cases, slightly increased surface tension values at $\mathrm{CMC}_{\text {system }}$ are obtained compared to those for the single surfactants at their CMC values. Figure 7 shows the solubilization curves of liposome suspensions (lipid concentration $0.5 \mathrm{mM}$ and PCIPA molar ratio 9:1) due to the addition of different amounts of D-Bet, SDS, SDES and OP-10EO, respectively. Comparison of Figures 6 and 7 shows that the $\mathrm{CMC}_{\text {system }}$ values corresponded in all cases to the $\mathbf{S}_{\text {sat }}$ parameters, i.e., the surfactant.concentrations producing bilayer saturation of these systems. The results for lipid compositions PC/PA 9:1 and 8:2 molar ratios are given in Table 2.

In light of this agreement and bearing in mind that Lichtenberg (5)postulated the solubilization of liposomes by surfactants via the formation of mixed micelles, we can assume that the $\mathrm{CMC}_{\mathrm{system}}$ parameter corresponds to the $\mathrm{CMC}$ of mixed micelle formation during the solubilizing process. Lichtenberg, in his review (5), expresses the need 


\section{TABLE 2}

Surfactant Concentrations Corresponding to the $\mathbf{S}_{\mathrm{s} a t}$ Parameter of Liposomes (PCIPA 9:1 and 8:2 molar ratios, $0.5 \mathrm{mM}$ lipid concentrations) and $\mathbf{C M C}_{\text {system }}$ : Bilayer Lipid Composition a

\begin{tabular}{lccccc}
\hline & \multicolumn{2}{c}{ PC/PA (9:1) } & & \multicolumn{2}{c}{ PC/PA (8:2) } \\
\cline { 2 - 3 } & $\begin{array}{c}\mathrm{S}_{\text {sat }} \\
(\mathrm{mM})\end{array}$ & $\begin{array}{c}\mathrm{CMC}_{\text {system }} \\
(\mathrm{mM})\end{array}$ & & $\begin{array}{c}\mathrm{S}_{\text {sat }} \\
(\mathrm{mM})\end{array}$ & $\begin{array}{c}\mathrm{CMC}_{\text {system }} \\
(\mathrm{mM})\end{array}$ \\
\hline D-Bet & 1.55 & 1.56 & & 1.50 & 1.50 \\
SDS & 1.08 & 1.04 & & 1.15 & 1.13 \\
SDES & 0.67 & 0.68 & & 0.75 & 0.74 \\
OP-10EO & 0.48 & 0.47 & & 0.51 & 0.52 \\
\hline
\end{tabular}

${ }^{a}$ See Table 1 and Figure 2 for abbreviations.

for experimental data to correlate the surfactant CMC with its solubilizing power. We have now obtained some experimental evidence in this area. From our results, we conclude that, in solubilizing processes of liposomes by different surfactants, the aqueous concentration of surfactant $\left(\mathbf{S}_{\mathrm{a}}, \mathbf{S}_{\mathrm{b}}\right.$ and $\left.\mathbf{S}_{\mathrm{c}}\right)$ is always similar or higher than the corresponding CMC value. The most striking result is that the Re is inversely correlated with the CMC of the surfactants tested, regardless of the negative charge of the liposomes. However, an exception has been detected for the nonionic surfactant OP-10EO, which has a small CMC value and appears to be particularly effective in bilayer saturation and solubilization. Moreover, in liposomelsurfactant interaction processes, the physicochemical parameter $\mathbf{C M C}_{\text {system }}$ can be regarded as an interesting molar ratio, capable of shedciing light on the solubilizing capacity of surfactants.

In this connection, we suggest that liposome solubilization by surfactants should be studied, not only taking into account the possible correlation with the CMC of the pure surfactants but also the more specific physicochemical properties of the new mixed micelles formed between phospholipids present in the bilayer and the surfactants during the solubilizing processes.

\section{ACKNOWLEDGMENTS}

This work was supported in part by funds from DGICYT (Dirección General de Investigación Cientifica y Técnica), Spain. The authors acknowledge the expert technical assistance of G. von Knorring.

\section{REFERENCES}

1. Ollivon, M., O. Eidelman, R. Blumethal and A. Water, Biochemistry 221695 (1988).

2. Paternostre, M.T., M. Roux and J.L. Rigaud, Ibid 222668 (1988).
3. Ruiz, J., F.M. Gofii and A. Alonso, Biochim. Biophys. Acta 932127 (1988).

4. Schubert, R., K. Beyer, H. Wolburg and K.HY. Schmidt, Biochemistry 25:5263 (1986).

5. Lichtenberg, D., Biochim. Biophys. Acta 821:470 (1985).

6. De la Maza, A., J. Sanchez Leal, J.L. Parra, M.T. Garcia and I. Ribosa, J. Am. Oil Chem. Soc. 68:315 (1991).

7. Gofii, F.M., M.A. Urbaneja, J.L.R. Arrondo, A. Alonso, A.A. Durrani and D. Chapman, Eur. J. Biochem. 160:659 (1986).

8. Urbaneja, M.A., A. Alonso, J.M. Gonzalez-Mafias, F.M. Gofii, M.A. Partearroyo, M. Tribout and S. Paredes, Biochem. J. 270:305 (1990).

9. Lichtenberg, D., R.J. Robson and E.A. Dennis, Biochim. Biophys. Acta 737:285 (1983).

10. Alonso, A., M.A. Urbaneja, F.M. Gofii, F.G. Carmona, F.G. Cánovas and J. Gomez-Fernandez, Ibid. 902:237 (1987).

11. Allen T.M., in Liposome Technology, Vol I, edited by G. Gregoriadis, CRC Press, Boca Raton, 1986, pp. 110-120.

12. Ribosa, I., M.T. Garcia, A. De la Maza and C. Trullás, Proc. XX Meeting C.E.D., 1989, pp. 399-418.

13. Idson B., in Surfactants in Cosmetics, Surfactant Science Series, Vol. 16, edited by Martin M. Rieger, Marcel Dekker Inc. 1985, Chapter 1.

14. Rosen, M.J., J. Colloid and Interface Sci. 79:587 (1981).

15. Singleton, W.S., M.S. Gray, M.L. Brown and J.L. White, J. Am. Oil. Chem. Soc. 42:53 (1965).

16. Rigaud, J.L., A. Bluzat and S. Buschlen, in Physical Chemistry of Transmembrane Ion Motion, edited by G. Spach, Elsevier Amsterdam, 1983, pp. 457-464.

17. Rigaud, J.L., A. Bluzat and S. Buschlen, Biochem. Biophys. Res. Commun. 111:373 (1983).

18. Szoka, F., and D. Papahadjopoulos, in Liposomes: Preparation and Characterization edited by C.G. Knight, Elsevier, Amsterdam, 1981, Chapter 3.

19. Szoka, F., F. Olson, T. Heath, W. Vail, E. Mayer and D. Papahadjopoulos, Biochim. Biophys. Acta 601:559 (1980).

20. Standard Methods. 14th, edited by Am. Public Health Association, Washington, D.C., 1976, pp. 466-484.

21. Lunkenheimer, K., and D. Wantke, Colloid and Polymer Sci. 259:354 (1981).

22. Rosen, M.L., in Surfactants and Interfacial Phenomena, edited by John Wiley and Sons, New York, 1978, p. 23.

23. Shore, S., and D.R. Berger, in Anionic Surfactants, Surfactant Science Series, Vol. 7, part I, edited by Warner M. Linfield, Marcel Dekker Inc., New York, 1976, Chapter 5.

24. Barker, G., in Surfactants in Cosmetics, Surfactant Science Series, Vol. 16, edited by Martin M. Rieger, Marcel Dekker Inc., New York, 1985, Chapter 9.

[Received July 31, 1992; accepted April 23, 1993] 